\title{
Numerical Analysis for Heat and Mass Transfer of Pure Vapour Condensation in Inclined Flat Finned Tube
}

\author{
DENG Hui ${ }^{\mathrm{a}}$, BAI Yan, WU Sai \\ The School of Control and Computer Engineering, North China Electric Power University, Changping \\ District, Beijing 102206, China \\ aemail: auto_dh@ncepu.edu.cn
}

Keywords: condensation; pure water vapour; inclined flat tube; numerical simulation

\begin{abstract}
A 3D mathematical model to the condensation of pure vapor flowing downward in an inclined flat finned tube is proposed and implemented numerically in Wolfram Mathematica ${ }^{\circledR}$. The predicted values for condensate rate and mean condensation HTC(Heat Transfer Coefficient) are in good agreement with the experimental data from a prototype tube(length: $2600 \mathrm{~mm}$, altitude: 50mm,width: $3 \mathrm{~mm}$, incline angel: $60^{\circ}$ ). The deviation is less than $15 \%$. Using the numerical model to calculate the local film thickness and local condensation HTC on the tube plate, it is found that the gravity makes dominant effect on processes of the condensation.
\end{abstract}

\section{Introduction}

Pure steam condensation in a compact flat finned tube has been increasingly applied for the direct air cooling condenser in fossil power plants, because it achieves much higher heat transfer coefficients. In such condensers, the hydraulic diameter of the flow channels formed between two flats is $20 \mathrm{~mm}$, with $250 \mathrm{~mm}$ in the altitude, and the channels are inclined to the vertical in $60^{\circ}$. The fundamental mechanisms of heat and mass transfer as well as of two-phase flow in these small channels are not well understood.

Nusselt [1], in 1916, first presented a theoretical model for laminar film condensation on a vertical flat plate. In the analysis, the liquid film was assumed to laminar and the interfacial shear stress between the liquid and vapor was neglected. Spendel [2] studied theoretically the condensation inside vertical two phase closed thermosyphons taking into account the effect of interfacial shear stress and vapour pressure variation. The analytical results of local and average condensation coefficients differed from those predicted by Nusselt's theory by about 24 to11 \% and about $1-4 \%$, respectively. Fiedler [3][4] investigated experimentally the reflux condensation of refrigerant R134a in an inclined small diameter tube. It is found that the inclination angle has a significant effect on the heat transfer coefficient. Kim [5] presented a physical model for calculating the liquid film thickness and condensation heat transfer coefficient in a vertical condenser tube. The results demonstrated that the model described reasonably well the liquid film thinning effect by the vapor shear.

In this study, the condensation of water vapour is investigated in a single sub-channel of a prototype condenser [6], namely an inclined flat finned tube. The inner diameter of the test tube is 3 $\mathrm{mm}$ in width, $50 \mathrm{~mm}$ in altitude and its axial length is $2600 \mathrm{~mm}$.

\section{Physical model description}

Fig. 1 shows a differential control volume of the condensate film inside the tube that is inclined at an angle $\beta$ to the vertical. The control volume is acted upon by the force of gravity in altitude and in axial direction, i.e. in $x$ - direction and $z$ - direction respectively. The surface temperature of the inner tube wall is $T_{\mathrm{w}}$. The condensate film at the phase interface is at the saturation temperature $T_{\mathrm{s}}$. Consequently the condensate film is drained on the wall at the axial and altitude direction of the tube. 


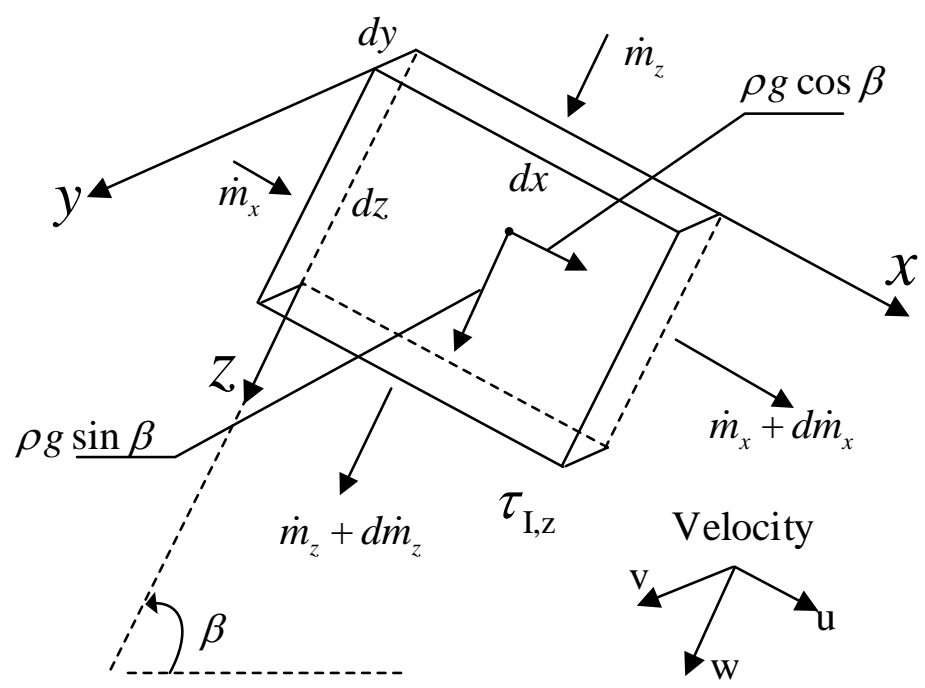

Fig.1. Physical model of film condensation in an inclined flat tube

Assuming a steady-state flow, the following differential equations for the velocity components in $x$ - and $z$ - direction are obtained from a balance of forces acting on a differential control volume of the condensate film (Fig.1) according to the classical Nusselt [1] film condensation theory:

$\mu \frac{\partial^{2} u}{\partial y^{2}}+\left(\rho-\rho_{v}\right) g \cos \beta=0$
$\mu \frac{\partial^{2} w}{\partial y^{2}}+\left(\rho-\rho_{v}\right) g \sin \beta=0$

An integration yields the velocity profiles in $x$ - and $z$ - direction:

$$
\begin{aligned}
& u=\frac{\left(\rho-\rho_{v}\right)}{\mu}\left(\delta y-\frac{\delta^{2}}{2}\right) \mathrm{g} \cos \beta \\
& w=\frac{\left(\rho-\rho_{v}\right)}{\mu}\left(\delta y-\frac{\delta^{2}}{2}\right) \mathrm{g} \sin \beta
\end{aligned}
$$

The mean velocities are obtained by an integration across the film thickness:

$$
\begin{aligned}
& u_{\mathrm{m}}=\frac{1}{\delta} \int_{0}^{\delta} u(y) d y=\frac{\left(\rho-\rho_{v}\right) \mathrm{g} \cos \beta}{3 \mu} \delta^{2} \\
& w_{\mathrm{m}}=\frac{1}{\delta} \int_{0}^{\delta} w(y) d y=\frac{\left(\rho-\rho_{v}\right) \mathrm{g} \sin \beta}{3 \mu} \delta^{2}
\end{aligned}
$$

The mass flow rate in $x$ - and $z$ - direction can be expressed in terms of the mean velocities as follows:

$$
\begin{aligned}
& \dot{m}_{\mathrm{x}}=u_{\mathrm{m}} \rho \delta \mathrm{dz} \\
& \dot{m}_{\mathrm{z}}=w_{\mathrm{m}} \rho \delta \mathrm{dx}
\end{aligned}
$$

By formation of the condensate mass flow rate, $d \dot{M}_{\text {con }}$, the heat transfer rate

$$
d \dot{Q}=\lambda_{\mathrm{v}} d \dot{M}_{\text {con }}
$$

is liberated. Assuming that this heat transfer rate is transferred by pure heat conduction across the condensate film, thus the heat rate transferred in a surface element $d x d z$ is:

$$
d \dot{Q}=\frac{\kappa\left(T_{\mathrm{s}}-T_{\mathrm{w}}\right)}{\delta} d x d z
$$

From Eqs. (9) and (10) follows:

$$
\lambda_{\mathrm{v}} d \dot{M}_{\text {con }}=\frac{\kappa\left(T_{\mathrm{s}}-T_{\mathrm{w}}\right)}{\delta} d x d z
$$


The vapour condensing on a surface element causes an increase of the condensate mass flow rate in altitude direction ( $x$ - direction) as well as in axial direction (z-direction). Therefore Eq. (11) can be written as:

$$
\lambda_{\mathrm{v}}\left(d \dot{m}_{\mathrm{x}}+d \dot{m}_{\mathrm{z}}\right)=\frac{\kappa\left(T_{\mathrm{s}}-T_{\mathrm{w}}\right)}{\delta} d x d z
$$

By combining Eqs. (5) - (8) with Eq. (12) the following nolinear PDE(partial differential equation) for the local film thickness $\delta(x, z)$ is obtained:

$$
\delta^{3}\left(\cos \beta \frac{\partial \delta}{\partial x}+\sin \beta \frac{\partial \delta}{\partial z}\right)=\frac{\mu \kappa\left(T_{\mathrm{s}}-T_{\mathrm{w}}\right)}{\lambda \rho\left(\rho-\rho_{\mathrm{v}}\right) \mathrm{g}}
$$

With the abbreviations

$$
\psi=\frac{\lambda \rho\left(\rho-\rho_{\mathrm{v}}\right) \mathrm{g} \delta^{4}}{\mu \kappa}
$$

the nolinear PDE, Eq. (15), can be converted to the form of linear PDE as follows:

$$
\cos \beta \frac{\partial \psi}{\partial x}+\sin \beta \frac{\partial \psi}{\partial z}=4\left(T_{I}-T_{w}\right)
$$

Eq. (15) is solved numerically in Wolfram Mathematica ${ }^{\circledR}$.

\section{Model validation}

The proposed model is used to predict the condensation mass flow rate and mean condensation HTC of a prototype condenser tube using the same dimension and same operating conditions as that used in the experimental condenser of Du Xiaoze [6].

The overall condensation mass flow rate of the condenser is obtained by accumulating the condensation mass rates drained from all sub-tube of the condenser, as follows:

$$
\dot{M}_{\text {con }}=310 \times\left(2 \int_{0}^{0.05} d \dot{m}_{\mathrm{z}}+2 \int_{0}^{2.6} d \dot{m}_{\mathrm{x}}\right)
$$

The mean condensation HTC of the whole condenser, $h_{\mathrm{c}}$, is represented by formation of that defined in a single condensation tube neglecting the heat transfer along the axial length, as follows :

$$
h_{\mathrm{c}, \mathrm{m}}=\frac{1}{2.6} \int_{0}^{z}\left(\frac{1}{0.05} \int_{0}^{0.05} \frac{\kappa}{\delta} d x\right) d z
$$

\begin{tabular}{|c|c|c|c|c|c|c|}
\hline \multirow{2}{*}{$\begin{array}{l}\text { Air volume } \\
\text { /(m3/s) }\end{array}$} & \multirow{2}{*}{$\begin{array}{c}\text { Air temperature } \\
/(\mathrm{K})\end{array}$} & \multirow{2}{*}{$\begin{array}{l}\text { Vapour mass } \\
/(\mathrm{t} / \mathrm{h})\end{array}$} & \multirow{2}{*}{$\begin{array}{l}\text { Vapour temperature } \\
\qquad /(\mathrm{K})\end{array}$} & \multicolumn{2}{|c|}{ Condensate rate/(t/h) } & \multirow{2}{*}{$\begin{array}{l}\text { Error } \\
/(\%)\end{array}$} \\
\hline & & & & experiment result & simulation solution & \\
\hline 5.2 & 306.55 & 0.69 & 373.85 & 0.639 & 0.653 & 2.19 \\
\hline 5.1 & 306.65 & 0.60 & 374.15 & 0.566 & 0.585 & 3.36 \\
\hline 5.1 & 307.15 & 0.58 & 373.85 & 0.545 & 0.562 & 3.12 \\
\hline 4.9 & 306.85 & 0.60 & 374.15 & 0.540 & 0.558 & 3.33 \\
\hline 4.8 & 305.95 & 0.60 & 374.05 & 0.540 & 0.558 & 3.33 \\
\hline 4.8 & 305.45 & 0.61 & 373.85 & 0.517 & 0.558 & 7.93 \\
\hline 4.8 & 306.55 & 0.60 & 373.85 & 0.511 & 0.558 & 9.20 \\
\hline
\end{tabular}

Tab. 1 shows comparisons of condensation mass flow rate between the numerial simulation results and the experimental results of Du Xiaoze [6] for the runs listed in Table 1. The simulation and the experimental results are in good agreement. Except for the last two runs the deviation between model and experiment is less than $10 \%$.

Tab.1 Comparison of condensation rate between numerical results and the experimental results of Du Xiaoze[16] runs listed in Table.1

Fig.2 demonstrates the comparison of the mean condensation HTC between the numerial simulation results and the experimental results of Du Xiaoze [6] for the runs listed in Table 3. The simulation results in general agree well with the directly measured quantities within a margin of error of $15 \%$. 


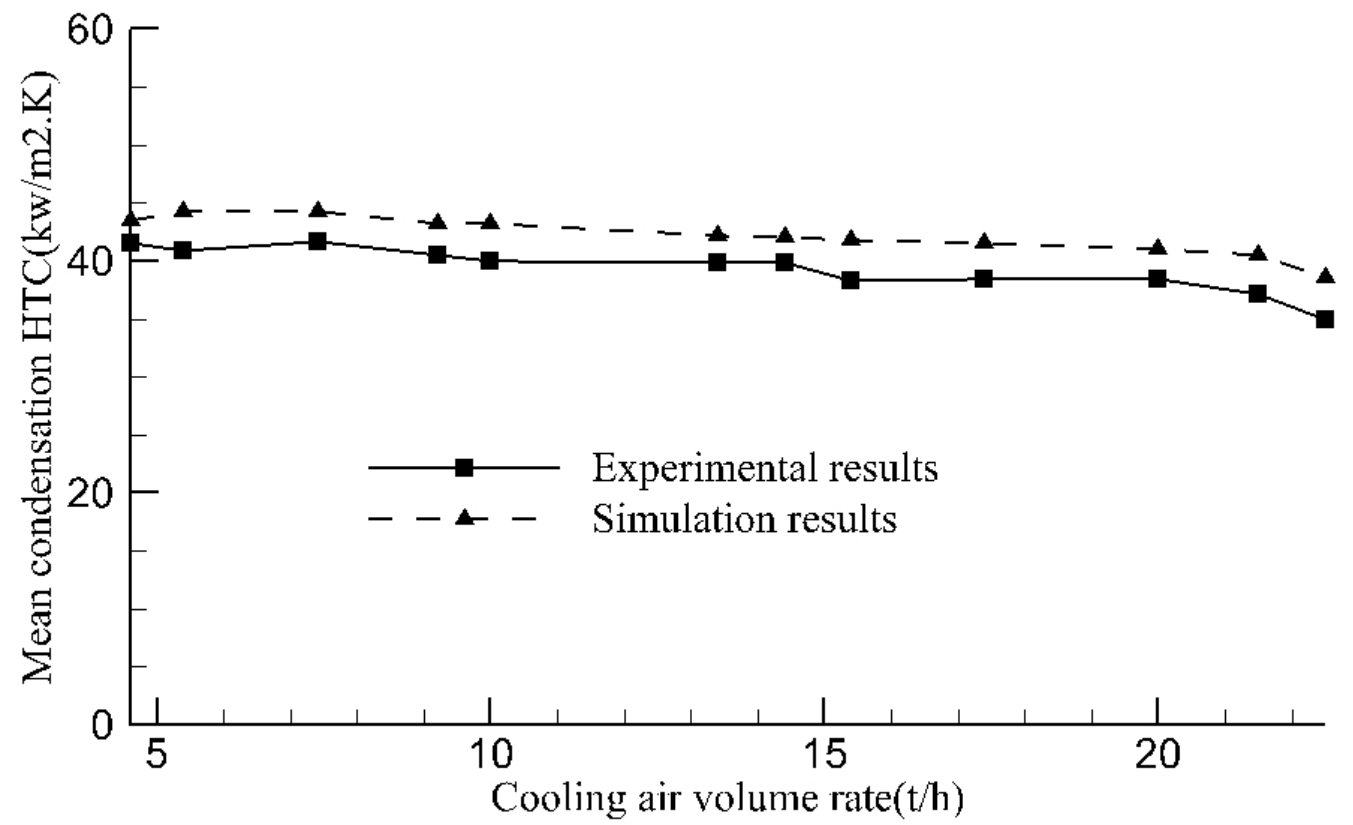

Fig.2. Comparison of condensation HTC between numerical results and the experimental results of Du Xiaoze ${ }^{[16]}$ runs listed in Table.3

\section{Analysis on heat and mass transfer}

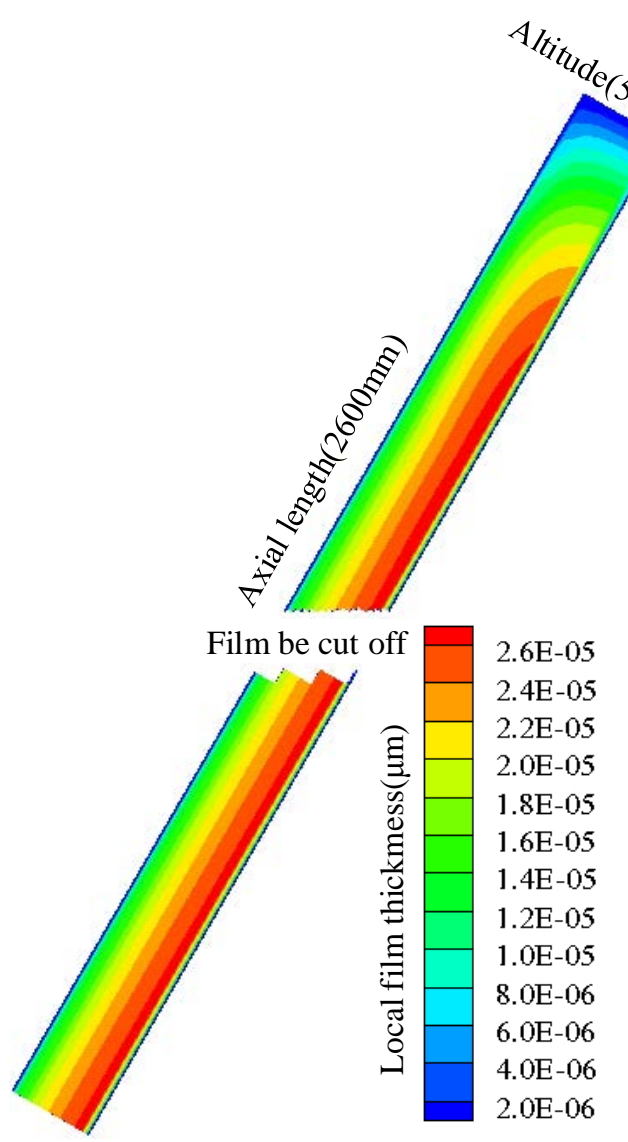

(a)

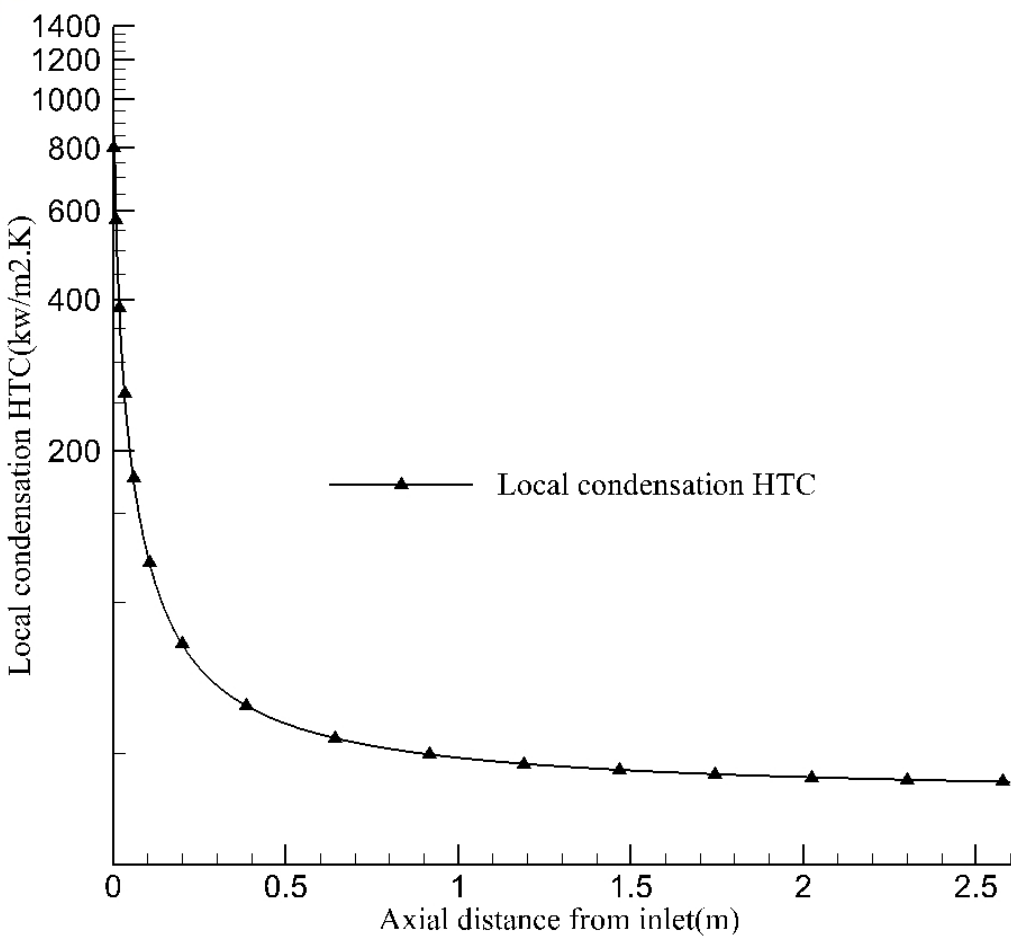

(b)

Fig.3 Numerical simulation to heat and mass transfer in condensation on plate wall

(a) Flood drawing of local film thickness on plate; (b) Local HTC vs. axial length in line-log scale The local liquid film thickness on the plate predicted by the proposed model is drawn in flood(Fig 3a). The film thickness behaves reasonably stable in most sections on the tube plate, whereas it 
increases significantly in the region near the tube inlet. Fig. 3a reflectes the gravitational effect on the film appropriately.

Fig. 3b shows that the local condensation HTC drops rapidly at first, and then decreases slowly as the axial distance from the entrance increases. The condensation HTC decrease with increase in film thickness and impact of film thickness is significant at the entrance region where condensation is efficient.

Comparing the Fig. 3a and Fig. 3b, at the region near to the inlet of the tube, the liquid film is very thin, condensation is efficient due to the small heat transfer resistance. As a result, high heat flux, condensation HTC and condensation mass flow can be reached. With the continuous condensation of steam and gravity accumulating the film along the condenser tube length, the film thickness increases rapidly so that the heat transfer resistance is larger. Ater the increased film thickness being in equilibrium with gravity accelerating effect, the film thickness and local condensation keep steady in the tube axial direction.

\section{Conclusion}

A 3D analytical model was used to study the heat and mass transfer for steam condensation in the inclined flat finned tube. The model predicted results agree well with the experimental results.

The results from model also showed that the condensate film thickness increased along condenser tube length. Local condensation HTC decreased with condenser tube length and the condensation HTC drops sharply at the entrance indicating the entrance effect.

\section{References}

[1] Nusselt W. Die Oberflachenkondesation des Wasserdamffes the surface condensation of water, J. Zetrschr. Ver. Deutch. Ing., 1916, 60: 541-546. $\mu$

[2] Spendel T. Laminar film condensation heat transfer in closed two-phase thermosyphons. In: Reay DA, editor. Advances in heat pipe technology. Oxford: Pergamon Press, 1981: 163-73.

[3] S. Fiedler, H. Auracher H. Experimental and theoretical investigation of reflux condensation in an inclined small diameter tube, J. International journal of heat and mass transfer, 2004, 47(19): 4031-4043.

[4] S. Fiedler, H. Auracher. Pressure drop during reflux condensation of R134a in a small diameter tube, in: Proc. Int. Symp. Compact Heat Exchangers, Grenoble, France, 2002: 369 - 373.

[5] Kim D E, Yang K H, Hwang K W, Ha Y H, Kim M H. Pure steam condensation model with laminar film in a vertical tube, J. International Journal of Multiphase Flow, 2011, 37(8): 941-946.

[6] Du Xiaoze, Yang Lijun, Jin Yansheng, Jiang Jianbo, Yang Yongping. Development of experimental correlation for heat transfer coefficient of direct air-cooled condenser in power plant, J. Proceedings of the CSEE, 2008, 28(14): 32-37. 